\title{
A decisão de ser médico: estudo multicultural Brasil-Portugal
}

\section{The decision to be a physician: a Brazil-Portugal multicultural study}

\author{
Silvia de Melo Cunha' (D) | silviamelo@unifor.br \\ Ana Maria Fontenelle Catrib' ${ }^{1}$ (D) catrib@unifor.br \\ Aline Veras Morais Brilhante' (D) alineveras@unifor.br \\ Elaine Saraiva Feitosa' (1) elainesfeitosa@gmail.com \\ Maria Amélia Duarte Ferreira² (D) mameliaferreira@med.up.pt
}

\section{RESUMO}

Introdução: A definição de uma carreira profissional representa a primeira grande decisão do adolescente e, em geral, ocorre num contexto de indecisões, conflitos e transformações, típicos dessa fase da vida.

Objetivo: Este estudo teve como objetivo conhecer as motivações que levam adolescentes a escolher o curso de Medicina no Brasil e em Portugal. Método: Trata-se de estudo de abordagem qualitativa, do tipo exploratório, que utilizou entrevistas abertas. Participaram 17 portugueses e 14 brasileiros, ingressantes no curso de Medicina, de uma universidade pública do Norte de Portugal e de uma universidade privada do Nordeste do Brasil, no ano de 2018. Para análise e interpretação das falas, utilizou-se a análise de conteúdo de Bardin, na modalidade temática.

Resultado: Identificaram-se duas temáticas - motivações intrínsecas e motivações extrínsecas - e seis núcleos de sentido: disposição para ajudar os outros, tendência natural, medicina como ciência, interesse pela pesquisa, influência de modelos e estabilidade profissional/ status social.

Conclusão: Os adolescentes ingressantes em Medicina no Brasil e em Portugal apresentaram similaridade na motivação para a escolha profissional. Prevaleceram as motivações intrínsecas, e a disposição para ajudar os outros foi a razão mais frequente para escolher Medicina nos dois países.

Palavras-chave: Adolescência; Escolha Profissional; Motivação; Medicina; Educação Médica.

\section{ABSTRACT}

Introduction: The definition of a professional career represents the adolescent's first major decision and, in general, it occurs in a context of indecision, conflicts and transformations, typical of this stage of life.

Objective: To understand the motivations that lead adolescents to choose a medical career in Brazil and in Portugal.

Method: Exploratory study with a qualitative approach, which used open interviews. The participants were 17 Portuguese and 14 Brazilian freshman medical students attending a public university in the north of Portugal and a private university in the northeast of Brazil in 2018. Bardin's Content Analysis was used for the analysis and interpretation of the speeches, using the thematic modality.

Result: Two thematic modalities were identified: intrinsic and extrinsic motivations and six cores of meaning: willingness to help others, natural tendency, Medicine as a science, interest in research, influence of models, professional stability/social status.

Conclusion: Adolescent freshman medical students in Brazil and Portugal showed similar motivations for their professional choice. Intrinsic motivations predominated and the willingness to help others was the most frequent reason for choosing the medical course in both countries.

Keywords: Adolescence; Professional Choice; Motivation; Medicine; Medical Education.

${ }^{1}$ Universidade de Fortaleza, Fortaleza, Ceará, Brasil.

${ }^{2}$ Faculdade de Medicina da Universidade do Porto, Porto, Portugal.

Editora-chefe: Rosiane Viana Zuza Diniz. Editor associado: Danilo Borges Paulino.

Recebido em 22/06/21; Aceito em 23/10/21.

Avaliado pelo processo de double blind review. 


\section{INTRODUÇÃO}

A escolha da profissão é um processo contínuo que se desenvolve de modo não linear, influenciado por decisões tomadas ao longo da vida. Escolher uma profissão é decidir o futuro, o que fazer, o modo de viver, e, acima de tudo, é escolher sobre quem ser e quem não ser $^{1-3}$.

A definição de uma carreira profissional representa a primeira grande decisão do adolescente e, em geral, ocorre num contexto de indecisões, conflitos e transformações, típicos dessa fase da vida, o que pode tornar essa escolha um momento difícil e doloroso ${ }^{2,4-6}$.

A escolha profissional está intimamente relacionada ao conceito complexo de motivação, cujo sentido, oriundo do latim motivus e do verbo motivare, lembra movimento, podendo ser conceituada como aquilo que impulsiona - comportamento de um indivíduo para alcançar um determinado objetivo ${ }^{7,8}$. Estar motivado significa ser impulsionado para realizar alguma ação. Um mesmo indivíduo pode ter tipos e intensidades diferentes de motivação. Investigar o que motiva o comportamento humano é buscar compreender os porquês de suas ações ${ }^{8,9}$.

Numerosas teorias buscaram explicar motivação, mas nenhuma foi universalmente aceita ou capaz de abranger a complexidade da natureza humana e de seus aspectos comportamentais. A teoria mais citada nas publicações de educação médica é a da autodeterminação que reconhece dois tipos principais de motivação: extrínseca e intrínseca. Na motivação extrínseca, o que impulsiona são os resultados esperados, como recompensas ou punições, e, na intrínseca, o que move o indivíduo é algo inerentemente interessante ou agradável ${ }^{9-11}$.

A motivação é um preditor significativo de aprendizado, sucesso acadêmico e determinação de continuar estudando Medicina, e desempenha papel importante no desenvolvimento da identidade profissional ${ }^{10,12}$.

Decidir ser médico significa enfrentar desafios, e a motivação para essa escolha tem se mostrado fundamental. Uma escolha sem motivação pode predispor a uma abordagem superficial da aprendizagem e ao abandono da formação com graves prejuízos pessoais e profissionais ${ }^{10}$.

Medicina é uma profissão de prestígio no Brasil e em Portugal, onde mais da metade dos médicos trabalha nos serviços público e privado. No Brasil, 93\% dos graduados exercem integral e exclusivamente medicina, com remuneração diferenciada em relação aos outros profissionais da saúde. Em Portugal, onde o mercado de trabalho e a progressão na carreira médica requerem uma pós-graduação, o crescente déficit de vagas para especialização no Sistema Nacional de Saúde tem aumentado o número de médicos sem especialidade e reduzido as expectativas de ingresso num plano de carreira com remuneração progressiva ${ }^{13-15}$.

Historicamente próximos, os dois países têm algumas similaridades linguísticas e culturais e muitas diferenças econômicas, políticas e sociais. O intercâmbio de alunos de Medicina é frequente, e, na última década, avolumaram-se os pedidos de revalidação de diploma médico de brasileiros em Portugal.

A motivação para fazer Medicina tem sido estudada por diversos pesquisadores em diferentes países. Alguns estudos têm comparado os seus resultados em diferentes realidades sociais e culturais ${ }^{16,17}$. Há, entretanto, uma lacuna de estudos que versem sobre fatores motivacionais na escolha da carreira médica comparando Brasil e Portugal.

O conhecimento sobre a motivação para a escolha profissional dos alunos ingressantes possibilitará o planejamento do currículo e de atividades acadêmicas que favoreçam a aprendizagem, a adaptação do aluno na universidade e a construção de sua identidade profissional.

Nesse sentido, este estudo objetivou compreender as motivações que levam adolescentes a escolher o curso de Medicina no Brasil e em Portugal.

\section{MÉTODO}

Trata-se de estudo multicultural, exploratório, de natureza qualitativa, que buscou responder a questões do universo de significados, valores, atitudes e motivos que permeiam a percepção dos sujeitos da pesquisa, contemplando narrativas sobre sentimentos e opções de vida, compreendendo a complexidade do ser humano e a singularidade de suas reações no meio em que vive ${ }^{18,19}$.

Selecionaram-se 17 discentes de uma universidade portuguesa e 14 de uma universidade brasileira. Para participar da pesquisa, o indivíduo deveria estar matriculado no primeiro ano do curso de Medicina e ter idade inferior a 19 anos. Não houve definição prévia do número de participantes, sendo utilizado o critério de saturação das respostas para compreensão do fenômeno, objeto do estudo. Os discentes foram convidados em sala de aula, e aqueles que demonstraram interesse marcaram horário para a entrevista, assinaram o Termo de Consentimento Livre e Esclarecido (TCLE) e foram incluídos como participantes.

Os cursos de Medicina, nos quais se desenvolveu o estudo, apresentam importantes diferenças entre si. A Faculdade de Medicina da Universidade do Porto (FMUP), estudada em Portugal é centenária, o ingresso de seus alunos é anual, e o currículo disciplinar é composto por dois ciclos: ciclo de estudos básicos em ciências da vida (três primeiros anos) e ciclo de prática clínica que integra terapêutica médica, radiologia e anatomia patológica especial (três últimos anos). 
No Brasil, o curso de Medicina da Universidade de Fortaleza (UNIFOR) incluído no estudo foi criado em 2006, o ingresso é semestral, e o currículo é composto por módulos que integram longitudinalmente disciplinas de ciências básicas e prática clínica nos primeiros quatro anos, e o estágio supervisionado (internato médico) nos dois últimos anos.

A Tabela 1 apresenta uma resumida caracterização das instituições de ensino superior (IES) e dos participantes do estudo.

A coleta de dados ocorreu de fevereiro a abril de 2018 no Brasil, e em setembro do mesmo ano em Portugal. Utilizaramse entrevistas abertas, com a seguinte pergunta norteadora: "Por que você decidiu fazer Medicina?".

Com o objetivo de proteger a identidades dos discentes, adotou-se o seguinte critério: "I" (ingressantes), com "B" (Brasil) e "P" (Portugal), e números que representam cada um dos participantes de cada grupo.

As entrevistas transcritas foram lidas em profundidade e analisadas à luz da análise de conteúdo de Bardin ${ }^{20}$, na modalidade temática, que consiste em buscar os núcleos de sentido (NS) que compõem uma comunicação, seguindo-se as etapas de pré-análise, exploração do material, tratamento dos resultados e interpretação.

A análise temática iniciou-se com a leitura das falas, depoimentos, para, depois, aprofundar-se nos sentidos manifestados, relacionando os significantes (estruturas semânticas) com os significados (estruturas sociológicas) emergentes do material. Dessa forma, articularam-se as falas com as variáveis psicossociais, o contexto cultural e o processo de produção da mensagem.

Considerando que a teoria da autodeterminação é a mais citada para definir motivação nas publicações em educação médica, optou-se por definir previamente as temáticas deste estudo em motivação extrínseca e motivação intrínseca. $\mathrm{Na}$ motivação extrínseca, o que define a escolha são os resultados esperados, como recompensas ou punições, e, na motivação intrínseca, o que impulsiona o indivíduo é algo inerentemente interessante ou agradável ${ }^{9-11}$.

As temáticas e os NS que emergiram das falas dos participantes foram posteriormente cotejados com abordagens teóricas sobre escolha profissional.

O estudo foi aprovado pelo Comitê de Ética e Pesquisa em Seres Humanos da Universidade de Fortaleza (UNIFOR) com o número 3.954.616, estando em conformidade com os princípios éticos e as determinações da Resolução n 510/2016 do Conselho Nacional de Saúde (CNS). Em Portugal, foi aprovado pela Comissão de Ética da Faculdade de Medicina da Universidade do Porto (FMUP) e do Complexo Hospitalar São João.

\section{RESULTADOS E DISCUSSÃO}

A análise das respostas dos ingressantes entrevistados sobre a motivação para a escolha profissional partiu das ideias associadas presentes nos depoimentos, dos quais emergiram seis NS ou subcategorias temáticas que foram posteriormente agrupados em duas temáticas, apresentados na Tabela 2.

Tabela 1. Caracterização das universidades e dos estudantes de Medicina.

\begin{tabular}{lcc}
\hline \multicolumn{1}{c}{ CARACTERíSTICA } & UNIFOR & FMUP \\
\hline Natureza administrativa da instituição & PRIVADA & PÚBLICA \\
Número de ingressantes por ano & 200 & 245 \\
Metodologia de ensino e aprendizagem & Aprendizagem Baseada em Problemas & Tradicional \\
Carga horária total & 8.532 horas & 10.080 horas \\
Participantes do estudo & 14 & 17 \\
Sexo masculino & 4 & 4 \\
Sexo feminino & 10 & 13 \\
Média da idade dos participantes & 18,2 & 17,8 \\
\hline
\end{tabular}

Tabela 2. Motivação para a escolha do curso de Medicina no Brasil e em Portugal, 2018.

\begin{tabular}{cccc}
\hline TEMÁTICAS & NÚCLEOS DE SENTIDO & UNIFOR & FMUP \\
\hline \multirow{3}{*}{ Motivação intrínseca } & Disposição para ajudar os outros & 8 & 10 \\
& Tendência natural & 7 & 3 \\
& Medicina como ciência & 2 & 6 \\
\hline \multirow{2}{*}{ Motivação extrínseca } & Interesse pela pesquisa & 0 & 2 \\
& Influência de modelos & 4 & 2 \\
& Estabilidade profissional/status social & 2 & 6 \\
\hline
\end{tabular}


Para a maioria dos participantes deste estudo, a escolha profissional incluiu mais de um fator motivacional, caracterizando, algumas vezes, motivações intrínsecas e extrínsecas em um mesmo estudante. Os resultados demonstram claramente que a maioria dos entrevistados em Portugal e no Brasil são intrinsecamente motivados. Essa similaridade ocorre apesar das diferenças culturais, sociais e econômicas entre os dois países. Resultado semelhante foi encontrado em estudos que compararam Reino Unido com Espanha e com Gana ${ }^{16,17}$.

Estudos realizados em diferentes países, com abordagens metodológicas quantitativas e qualitativas, que avaliaram diferentes gerações de alunos de Medicina, em momentos diversos do curso, e também recém-graduados ${ }^{12,16,17,21-25}$ apresentaram resultados semelhantes com predominância de uma tendência motivacional inerente, fonte natural de aprendizado e realizações, o que foi considerado elemento crítico no desenvolvimento cognitivo, social e físico ${ }^{9}$. Indivíduos intrinsecamente motivados têm maior probabilidade de se envolver em um aprendizado mais profundo e demonstrar melhor desempenho, maior criatividade e crescimento de uma forte identidade profissional ${ }^{12}$.

O predomínio do comportamento intrinsecamente motivado aponta para a necessidade de metodologias de aprendizagem que estimulem a autonomia dos alunos e favoreçam o desenvolvimento de competências técnicas e de vida que contemplem suas expectativas altruísticas e vocacionais, garantindo seu melhor desempenho.

\section{Motivação intrínseca}

Neste estudo, a influência da motivação intrínseca na escolha da graduação em Medicina revelou-se nos seguintes NS: disposição para ajudar os outros, tendência natural e medicina como ciência.

A escolha da medicina como carreira profissional motivada por questões humanitárias, presente na maioria das publicações sobre o tema, revela-se no âmbito deste estudo como a "disposição para ajudar os outros", que emerge explícita e implicitamente de depoimentos emocionados de adolescentes que discorrem sobre a vontade de acolher, de cuidar e mesmo de transformar o mundo e fazer a diferença na vida das pessoas. Esteve presente na narrativa de dez portugueses e oito brasileiros entrevistados, sendo o motivo mais frequente para fazer Medicina. Algumas vezes relacionam essa disposição com vivências e princípios religiosos ou de doenças graves em familiares, mas, na maioria dos relatos, é simplesmente porque gostam de ajudar.

Se eu vou ficar aqui nessa terra, nesse planeta por um tempo finito, eu quero gastá-lo bem, e eu sinto que ajudar as pessoas é um tempo bem gasto (IP2).
Eu não consigo ser indiferente ao sofrimento das pessoas e sempre gostei de ajudar (IP3).

Pelo princípio religioso, eu sinto no meu coração que eu posso atuar nessa área de cuidar, amar e salvar vidas (IB4).

Eu sempre tive o sonho de mudar o mundo, a perspectiva dele e cuidar dos que precisam, acho que ser médica não é só cuidar da doença (IB9).

Um estudo recente realizado no Brasil analisou as motivações para a escolha profissional do médico em recémgraduados e encontrou uma prevalência para questões humanitárias, como "ajudar as pessoas", "fazer o bem" e o "relacionamento com o paciente"24. Na Finlândia, o "interesse pelas pessoas" foi o principal motivo para querer estudar Medicina em 20 anos de pesquisa realizada nos anos de 1988, 1998 e 2008, com graduados há dois e 11 anos $^{23}$. E esse motivo está explícito no relato da estudante IB2: "Eu escolhi Medicina exatamente pelas pessoas".

Estudos quantitativos têm corroborado a prevalência de razões altruístas e humanitárias em diferentes países, e alguns autores descrevem maior tendência do sexo feminino em direção a interesses sociais e altruístas ${ }^{16,21,24}$. Neste estudo, entrevistaram-se oito ingressantes do sexo masculino, quatro portugueses e quatro brasileiros, e observou-se que a disposição para ajudar foi contemplada no discurso de metade dos acadêmicos. Os demais apontaram motivações extrínsecas para a escolha profissional.

A "tendência natural", expressa em grande parte dos relatos como uma inexplicável decisão tomada desde a mais tenra infância, citada por sete brasileiras, três portuguesas e nenhum participante do sexo masculino, foi a segunda motivação mais frequente no grupo do Brasil e a quarta no grupo de Portugal.

Implícita na resposta de quem não consegue imaginar a possibilidade de uma outra escolha, essa tendência ou inclinação natural tem sido descrita por diversos autores como "vocação", do latim vocatiōne, cuja origem do verbo vocare significa "chamar", sendo descrita como propensão, inclinação para qualquer atividade, ofício ou profissão ${ }^{26}$.

Eu nunca me vi a ser outra coisa desde pequenina (IP1).

Lembro de ter 5 anos e passar em frente ao Hospital São João e dizer que é pra aqui que eu quero vir. Foi um sonho desde sempre (IP17).

Eu sempre quis ser médica desde pequenininha (IB6).

Porque desde criança eu não me via fazendo outra coisa (IB12).

Um estudo realizado em Zaragoza com alunos do segundo ano de graduação (75\% de mulheres e $25 \%$ de 
homens) evidenciou que cerca de $60 \%$ dos pesquisados pensaram em medicina antes dos 14 anos, 40\% decidiram fazer o curso antes dos 16, e 50\% consideraram ser essa a única carreira que poderiam ter escolhido. Os participantes que pensaram e decidiram mais cedo são os que em maior porcentagem manifestaram ser a medicina a única carreira que poderia satisfazê-los. Esses resultados sugerem um forte componente vocacional, provavelmente reforçado pelo percentual de mulheres (75\%) na amostra ${ }^{21}$.

Um estudo finlandês, que reuniu grande amostra ao longo de 20 anos, demonstrou que a importância relativa da "vocação" aumentou no decorrer do tempo, sendo significativamente mais importante para as mulheres do que para os homens e figurando como elemento preditor de satisfação com a profissão médica. Um estudo realizado na Universidade Estadual do Rio Grande do Norte questionou seus alunos sobre a motivação para estudar Medicina e obteve como resposta mais frequente aptidões pessoais e vocacionais, que também foram relacionadas com maior satisfação com o curso e melhor desempenho acadêmico ${ }^{23,27}$.

Na Pensilvânia, o estudo realizado por Wrzesniewski et al. ${ }^{28}$ apresentou evidências que indicam uma maior satisfação com a vida e com o trabalho para os entrevistados que consideram seu trabalho um "chamado", mesmo quando a renda, a educação e a ocupação foram controlados.

O interesse científico pela medicina e a curiosidade no funcionamento do corpo humano são as ideias associadas que definem o NS "medicina como ciência", que representou a segunda motivação mais frequente no grupo de Portugal e a quarta no grupo do Brasil, sendo citado por seis portugueses e dois brasileiros neste estudo.

Gostava muito dos conhecimentos que poderia arrecadar (IP4).

Inicialmente me apaixonei pelo corpo humano, como é que isto funciona, como é que isto é possível (IP15).

Eu me interesso muito por conhecer o corpo humano (IB1).

Um estudo qualitativo realizado na Holanda e pesquisas quantitativas feitas na Noruega, na Espanha e no Reino Unido descreveram resultados semelhantes ao grupo de portugueses, em que o interesse pela ciência e o desejo de ajudar os outros foram as principais razões para que os discentes optassem por seguir uma carreira médica ${ }^{16,17,25,29-31}$.

Notou-se aqui uma diferença entre os grupos: somente dois brasileiros citaram o interesse em "conhecer o corpo humano" ou "na matéria", e sempre associado a outras motivações. Em outros estudos realizados no Brasil, não se encontrou a citação dessa categoria $22,24,27$.

\section{Motivação extrínseca}

A motivação extrínseca na escolha profissional, neste estudo, apresentou-se nos seguintes NS: estabilidade profissional/status social, interesse pela pesquisa e influência de modelos. A similaridade entre os grupos de portugueses e brasileiros, observada anteriormente, não foi mais explicitada, ficando evidentes as diferenças entre os grupos estudados nessa categoria.

Enquanto os ingressantes portugueses apontaram a estabilidade profissional/status social e o interesse pela pesquisa como prioridades da escolha, a motivação dos brasileiros concentrou-se na influência de modelos.

A "influência de modelos" revelou-se nas respostas dos adolescentes brasileiros participantes deste estudo, que apontaram a admiração por médicos, familiares ou não, como fator motivacional para exercer medicina. Em Portugal, ter um familiar médico foi mencionado por dois alunos. No Brasil, a escolha de "seguir os passos", a admiração por médicos e o "tentar se espelhar" denotam o papel relevante da influência de modelos na escolha profissional de quatro ingressantes.

Eu acho que tendo meu pai como médico que admiro, tomei isso pra seguir os passos dele (IB7).

Porque eu acho um trabalho muito bonito e tem alguns médicos que eu admiro muito e tento me espelhar (IB13).

Tive muita influência de um parente da família. Eu me inspirei muito nele (IB12).

Um estudo realizado na Espanha revelou que 70\% dos estudantes do segundo ano de Medicina afirmaram ter sido influenciados para a escolha profissional, e as influências mais frequentemente citadas foram: pressão familiar (16\%), contato com a doença (15\%), existência de familiares médicos (12\%), televisão (11\%), tutores (10\%) e amigos (5\%). Quando se avaliou o fator de influência somente no grupo de alunos que tinham parentes médicos, esse foi o principal fator influente (90\%), demonstrando que esse grupo tomou a decisão por si mesmo em percentual significativamente menor que o restante de alunos pesquisados ${ }^{21}$.

$\mathrm{Na}$ Holanda, um estudo qualitativo com alunos do ensino médio ressaltou que crescer em uma família médica aumentou a motivação dos alunos para estudar Medicina e facilitou o acesso à estágios na área da saúde, proporcionando vivências de assistência médica ${ }^{25}$.

O "interesse pela pesquisa" ou o desejo de ser investigador só esteve presente nas respostas dos portugueses, e esse resultado pode revelar, além de diferenças culturais sobre a importância da pesquisa em cada país, diferenças no perfil das duas universidades. 
Medicina era a opção que poderia me levar a fazer investigação mais facilmente, eu sempre sonhei em ser investigador (IP6).

Inicialmente eu estava indeciso entre dois cursos: Medicina ou Bioengenharia, mas o que eu queria mesmo era a investigação, e a medicina abre muitas outras portas, a investigação é muito melhor (IP16).

Nas duas últimas décadas, o interesse pela pesquisa tem sido apontado nos estudos sobre motivação realizados na Europa, o que provavelmente está associado às maiores oportunidades de financiamento por órgãos de fomento, bem como à diferença na carga horária destinada às atividades curriculares do curso ${ }^{16,17}$. Esse tipo de interesse não foi citado nos estudos brasileiros. Na universidade portuguesa estudada, a conclusão da graduação em Medicina está atrelada à defesa de uma dissertação e ao título de mestre, o que, por si só, já estimula a produção do conhecimento.

A presença da "estabilidade profissional/status social" como motivação para a escolha foi observada com a mesma frequência de "medicina como ciência" no grupo dos ingressantes portugueses, citada por seis entrevistados, e, no Brasil, emergiu em dois ingressantes. Esse NS revela-se nas entrelinhas das respostas de adolescentes ao tratarem a medicina como "possibilidade de garantir alguma estabilidade" ou "profissão mais nobre", mas também se apresenta de forma explícita na voz de quem afirma que as maiores oportunidades de emprego tiveram "um peso na escolha":

Escolhi medicina porque gosto da área da psicologia, mas a psicologia aqui em Portugal não tem muita saída, [...] assim tinha possibilidade de garantir alguma estabilidade, eu acho que foi mais ou menos isso, mas em termos de vocação não sei se seria o mais indicado pra mim (IP12).

As oportunidades de emprego na medicina acabam por ser maiores e ter um peso na escolha (IP14).

Eu decidi fazer Medicina porque eu tenho um grande interesse na área de saúde mental e decidi entrar pra Medicina porque acho que teria uma plataforma maior do que na psicologia (IB14).

Eu fazia enfermagem, e eu gostava, mas eu queria algo mais, eu não senti que eu tava completa e aqui eu sinto (IB11).

Alunosingressantesnosanos 2000e2006na Universidade Federal de Sergipe revelaram, como motivações para a escolha do curso de Medicina, o fecundo campo de trabalho, a alta empregabilidade e a remuneração diferenciada ${ }^{22}$.

O status social, a oportunidade de renda elevada e a segurança de empregabilidade foram descritos por Pastor et al. $^{16}$ como os fatores motivacionais menos escolhidos por estudantes no Reino Unido e na Espanha, sendo mais frequentes entre os homens ${ }^{17}$.

Para os finlandeses, "bom salário" e "profissão de prestígio" foram motivações frequentes para os homens. Chama a atenção, na série histórica de 20 anos, a redução da importância relativa da remuneração e das oportunidades de emprego como motivos de ingresso na carreira médica ${ }^{23}$.

Em estudo realizado no Brasil, o status social esteve presente e foi associado à importância da profissão médica na sociedade. O conhecimento adquirido e a atuação na assistência à saúde colocam o médico e mesmo o estudante de Medicina numa posição de destaque social ${ }^{22}$.

Para muitos pesquisadores, existe uma contradição no fato de os ingressantes de Medicina apontarem motivações altruístas e vocacionais para uma profissão que representa prestígio e poder ${ }^{27}$. De acordo com esses pesquisadores, o verdadeiro motivo dessa escolha profissional está relacionado à imagem de ascensão atrelada à Medicina, embora isso nem sempre seja verbalizado pelos estudantes. Segundo Trindade et al. ${ }^{22}$, o desejo expresso de ajudar os outros pode significar ser útil, importante e reconhecido socialmente como um médico humano, resultando em prestígio e status social.

\section{CONSIDERAÇÕES FINAIS}

Os resultados deste estudo contribuíram para demonstrar que, apesar das diferenças culturais e de modelos educativos distintos entre os dois países, os adolescentes ingressantes em Medicina estudados apresentaram algumas similaridades na motivação para a escolha profissional. Cada aluno é motivado por uma combinação de fatores, e, embora as motivações dos grupos tenham sido diferentes em termos de número de citações, prevaleceram as motivações intrínsecas nos dois grupos, e a disposição para ajudar os outros foi a razão mais frequente para escolher Medicina nos dois países.

Contudo, significativa diferença entre os estudantes portugueses e brasileiros foi demonstrada quando se avaliaram o interesse científico pela medicina e o interesse pela pesquisa (investigação). Esse resultado, provavelmente influenciado por questões culturais e pelo perfil institucional, deve servir de alerta para as implicações no comportamento do futuro profissional e sugere a necessidade de adequações curriculares na formação médica brasileira que incentivem a produção do conhecimento e condutas guiadas pelas melhores evidências científicas.

O maior percentual de mulheres nas escolas médicas tem sido associado ao maior número de participantes movidos por altruísmo e vocação. Esse resultado aponta ainda dois aspectos importantes: a possibilidade de a feminização na medicina estar imprimindo um caráter de humanização e o 
papel social no exercício da profissão. É importante ressaltar, entretanto, que altruísmo, vocação, humanização e papel social são frutos de construções sociais atribuídas ao feminino e não características "inatas" às mulheres.

As implicações futuras deste estudo apontam para duas direções. No campo da educação médica, este estudo estimula a utilização de metodologias de ensino e aprendizagem que contemplem as expectativas motivacionais e despertem o interesse científico dos alunos. No campo da pesquisa científica, desperta a necessidade de estudos que avaliem o impacto dos fatores motivacionais na performance acadêmica, no desenvolvimento da identidade profissional e na relação com a feminização na medicina.

\section{AGRADECIMENTOS}

Nossos agradecimentos à Universidade de Fortaleza (UNIFOR) e à Faculdade de Medicina da Universidade do Porto (FMUP) que nos permitiram realizar esta pesquisa, aos acadêmicos da FMUP e da UNIFOR que se dispuseram a participar do estudo e aos alunos bolsistas de iniciação científica da Unifor que se dedicaram a este estudo.

\section{CONTRIBUIÇÃO DAS AUTORAS}

Silvia de Melo Cunha e Aline Veras Morais Brilhante participaram da construção e do delineamento da pesquisa, da coleta, análise e interpretação dos dados, da redação do manuscrito e da revisão crítica do conteúdo intelectual relevante. Ana Maria Fontenelle Catrib participou da análise e interpretação dos dados, da redação do manuscrito e da revisão crítica do conteúdo intelectual relevante. Elaine Saraiva Feitosa e Maria Amélia Duarte Ferreira participaram da redação do manuscrito e da revisão crítica do conteúdo intelectual relevante.

\section{CONFLITO DE INTERESSES}

Declaramos não haver conflito de interesses.

\section{FINANCIAMENTO}

Declaramos não haver financiamento.

\section{REFERÊNCIAS}

1. Filomena K. Mitos familiares e escolha profissional: uma proposta de intervenção focada na escolha profissional à luz de conceitos da teoria sistêmica [dissertação]. Florianópolis: Universidade Federal de Santa Catarina; 2003.

2. Almeida FH, Silva LLM. Influência dos pais no processo de escolha profissional dos filhos: uma revisão da literatura. Psico USF. 2011;16(1):75-85.

3. Bohoslavsky R. Orientação vocacional: a estratégia clínica. 13a ed. São Paulo: Martins Fontes; 2015.

4. Lara LD, Araújo MCS. O adolescente e a escolha profissional: compreendendo o processo de decisão. Arq Ciências Saúde UNIPAR. 2005;9(1):57-61.
5. Gonzaga LRV, Lipp MEN. Relação entre escolha profissional, vocação e nível de estresse em estudantes do ensino médio. Psicol Argum. 2014;32(78):149-56

6. Silva JE, Fuzaro $\mathrm{CM}$, Pacheco MMDR. A escolha profissional para adolescentes: panorama de estudos e pesquisas. Rev Magistro. 2016;1(13):170-85.

7. Pedro AMD. Percursos e identidades. A (re)construção da identidade profissional do docente de enfermagem: olhar do docente [tese]. Lisboa: Universidade Lusófona de Humanidades e Tecnologias; 2011.

8. Wise RM. Motivação: teorias motivacionais do comportamento humano. Rev Cienc Gerenc. 2018;22(36):134-41.

9. Ryan RM, Deci EL. Intrinsic and extrinsic motivations: classic definitions and new directions. Contemp Educ Psychol. 2000;25(1):54-67.

10. Kusurkar RA, Cate TJT, Van Asperen M, Croiset G. Motivation as an independent and a dependent variable in medical education: a review of the literature. Med Teach. 2011;33(5):e242-62.

11. Lucena Filho EL. Motivação: confluências e divergências nas abordagens teórica. Rev Psicol Saberes. 2019;8(12):154-86.

12. Wasityasturi W, Susani YP, Prabandari YS, Rahayu GR. Correlation between academic motivation and professional identity in medical students in the Faculty of Medicine of the Universitas Gadjah Mada Indonesia. Educ Med. 2018;19(1):23-9

13. Scheffer MC, Guerra A, Guilloux AGA, Brandão APD, Miotto BA, Almeida CJ, et al. Demografia médica no Brasil 2020. São Paulo: FMUSP, CFM; 2020.

14. Santana $P$, Peixoto $H$, Duarte $N$. Demografia médica em Portugal: análise prospetiva. Acta Med Port. 2014;(27):246-51.

15. Santos SPM. A escola de medicina e a construção de uma identidade profissional [dissertação]. Porto: Universidade do Porto; 2019.

16. Pastor MA, López-Roig S, Sánchez S, Hart J, Johnston M, Dixon D. Analysing motivation to do medicine cross-culturally: the international motivation to do medicine scale. Escri Psicol. 2009;2(2):3-9.

17. Clayton B. Motivation for studying medicine: assessing the similarities between UK and Ghanaian medical student. Healthc Low Resour Settings. 2016;4(1):10-6.

18. Minayo MCS. O desafio do conhecimento: pesquisa qualitativa em saúde. 14a ed. São Paulo: Hucitec; 2014.

19. Jardim HG. Contemplar o adolescente no $3^{\circ}$ milénio. Funchal: Imprensa Académica; 2016.

20. Bardin L. Análise de conteúdo. São Paulo: Edições 70; 2016.

21. Soria M, Guerra M, Gimenez I. La decision de estudiar medicina: características. Educ Méd. 2006;9(2):91-7.

22. Trindade LMDF, Vieira MJ. Curso de Medicina: motivações e expectativas de estudantes iniciantes. Rev Bras Educ Med. 2009;33(4):542-54.

23. Heikklä T, Hyppölä $H$, Vänskä J, Aine $T$, Halila $H$, Kujala $S$, et al. Factors important in the choice of a medical career: a Finnish national study. BMC Med Educ. 2015 Oct;15:169.

24. Scheffer MC, Guilloux AGA, Dal Poz MR, Scharaiber LB. Motivos da escolha da profissão e perfil dos médicos recém-graduados no Brasil. Rev Assoc Med Bras. 2016;62(9):853-61.

25. Wouters A, Croiset G, Isik U, Kusurkar RA. Motivation of Dutch high school students from various backgrounds for applying to study medicine: a qualitative study. BMJ Open. 2017;7:e014779.

26. Michaelis dicionário escolar da língua portuguesa. 4a ed. São Paulo: Melhoramentos; 2016.

27. Cardoso Filho FAB, Magalhães JF, Silva KML, Pereira ISSD. Perfil do estudante de medicina da Universidade do Estado do Rio Grande do Norte (UERN), 2013. Rev Bras Educ Med. 2015;39(1):32-40.

28. Wrzesniewski A, McCauley C, Rozin P, Schwartz B. Jobs, careers, and callings: people's relations to their work. J Res Pers. 1997;31(1):21-33.

29. Vaglum P, Wiers-Jenssen J, Ekeberg O. Motivation for medical school: the relationship to gender and specialty preferences in a nationwide sample. Med Educ. 1999 Apr;33(4):236-42. 
30. Gasiorowski J, Rudowicz E, Safranow K. Motivation towards medical career choice and future career plans of polish medical students. Adv Health Sci Educ Theory Pract. 2015;20(3):709-25.
31. Gyorffy Z, Birkás E, Sándor I. Career motivation and burnout among medical students in Hungary - could altruism be a protection factor? BMC Med Educ. 2016;16:182. 